\title{
Simulation Analysis of Electric Power Control Model of Electric Vehicle
}

\section{Battery}

\author{
Li Ganglong ${ }^{1, a}$, Chen Yun ${ }^{1, a}$, Zhou Qing ${ }^{1, a}$, Hu Zhou ${ }^{1, a}$
}

${ }^{1}$ School of Mechanical Engineering, JiangXi University of Technology, Nanchang 330098,China
${ }^{a} 158926551 @ q q . c o m$

Keywords: power control, electric vehicle, model

\begin{abstract}
.
The electric control system of the electric vehicle battery is researched, a new fuzzy reasoning method is introduced, and the fuzzy PID controller is set up, the optimization and feedback idea are introduced in the this reasoning method, according to the different optimization indexes, the reasoning process is obtained, it can make the reasoning process be more effective. This paper gives the specific process of the reasoning method in application, it is used to establish model of power control system, and a PID controller is designed. The power control of electric vehicle battery is completed. In view of the power control process, the simulation is taken, the simulation result is analyzed, it shows that the method has good ability of power control, it can save the power effectively.
\end{abstract}

\section{Introduction}

With the worldwide energy shortage, the development of new energy vehicle has become a hot research topic in the field of automobile. Therefore, the electric car has become an inevitable trend of the automobile development. How to control the electric car battery power, this problem has become a hot research topic in the field of automobile. Because of the influence of complex factors of electric vehicle battery power storage capacity, power control mathematical model of battery electric vehicle is not possible to be built. According to this situation, the traditional control methods are often difficult to obtain satisfying control effect, the fuzzy control is developed in recent decades[1], it is a new control method. In the uncertain mathematical model and even unknown mathematical model, this control system shows unique superiority. In order to make the fuzzy control system has fast dynamic response, and has high steady state, PID control strategy is introduced in the fuzzy controller, fuzzy PI (or PID) composite control is obtained. At present, there are three kinds control methods are commonly used, such as hybrid fuzzy PID controller, switch fuzzy PID control structure, and the on-line adjustment fuzzy PID control system[2]. In this paper, the electric control system of the electric vehicle battery is researched, a new fuzzy reasoning method is introduced, and the fuzzy PID controller is set up, the optimization and feedback idea are introduced in the this reasoning method, according to the different optimization indexes, the reasoning process is obtained, it can make the reasoning process be more effective. This paper gives the specific process of the reasoning method in application, it is used to establish model of power control system, and a PID controller is designed. The power control of electric vehicle battery is completed. In view of the power control process, the simulation is taken, the simulation result is analyzed, it shows that the method has good ability of power control, it can save the power effectively[3,4]. 


\section{PID fuzzy control process of electric vehicle battery power and optimal fuzzy reasoning principle}

\section{A.Basic idea of optimal fuzzy reasoning}

For an electric vehicle battery power control system, assumed that the power control rules of the electric vehicle is credible, then fuzzy relation $\mathrm{R}$ is obtained based on the calculation of the inference antecedent and reasoning. It should be consistent with the corresponding fuzzy relation $\mathrm{R}$ rule, according to the given optimization index, then it is matching to J. Here, the match evaluation is embodied in the smallest distance if two fuzzy relations, in which fuzzy relation is represented by the fuzzy relation matrix. Distance is represented by the optimization index $J$, and $J$ is a function of R-R, in the premise of fuzzy relation matrix $\mathrm{R}$, corresponding to known reasoning component $\mathrm{A}$, with the optimization, the reasoning component $B$ can be computed, which makes $R=A \rightarrow B$, it is consistent to $\mathrm{R}$, the distance of given optimization index $\mathrm{J}$ is minimum[5].

If the $\mathrm{R}$ is consistent with $\mathrm{R}$, according to the index, the arbitrary optimized indexes are matching, so the above statements can also be shown as "consistent or the two given optimization indexes are consistent to J". It can be simplified as "according to the given optimization index J, consistent with most optimized index $\mathrm{J}$ ". Consistence is the most ideal target, it can meet the most matching beyond the reach of the case. It is not difficult to see that using optimal fuzzy reasoning method for electric vehicle batteries control, an inner loop (ring) is introduced, namely the reasoning component is driven and solved by R-R. Feedback ideas also are introduced in the optimal fuzzy reasoning. In this paper, the optimization index can be described as following formulas:

$$
\begin{aligned}
& J_{1}=\sum_{i=1}^{m} \sum_{j=1}^{n}\left|f\left(a_{i}, b_{j}\right)-r_{i j}\right| \\
& J_{2}=\sqrt{\sum_{i=1}^{m} \sum_{j=1}^{n}\left(f\left(a_{i}, b_{j}\right)-r_{i j}\right)^{2}}
\end{aligned}
$$

The basic idea of electric automobile battery power control meets the logical level requirements, here, the referred optimize index" is the "restrictions" of optimal fuzzy logic, such as the optimization index must be satisfied with the first rule constraints, at the same time, it meets the formulation about optimization problems of control, the closed loop is introduced. Thus, the introduction of optimal fuzzy reasoning has the basic of the above two aspects, therefore it has the certain feasibility.

\section{B. Optimal fuzzy reasoning method based on optimization index $J_{1}$}

The various elements $A=\left(a_{i}\right)_{m \times 1}$ are ordered from small to large, in accordance with the change of different lines of $\mathrm{A}$, the order of $\mathrm{B}$ rows are adjusted, thus, the new $A=\left(a_{i}\right)_{m \times 1}$ and $R=\left(r_{i j}\right)_{m \times n}$ are obtained, this adjustment does not change the value of the optimization index, and meet the following condition:

$$
\left.J_{1 j}=\sum_{i=1}^{m} \mid\left(a_{i} \wedge b_{j}\right)-r_{i j}\right)(j=1,2 \cdots, n)
$$


Analysis method is used to solve the value of $b_{j}^{*}$, make $0=a_{0} \leq a_{1} \leq \cdots \leq a_{m} \leq a_{m+1}=1$.

The strategy is: firstly, in accordance with the above sequence, range $[0,1]_{\text {of }} b_{j}$ is divides into

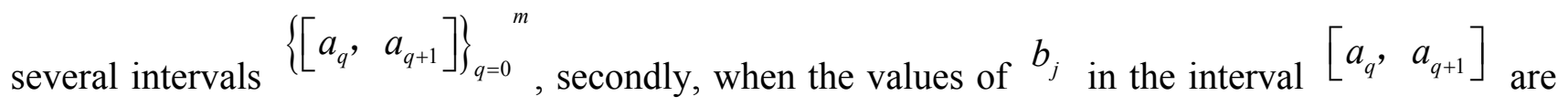
determined, according to the optimization function ${ }^{J_{1 \mathrm{j}}}$, the extreme value ${ }^{J_{1 \mathrm{j}}^{\mathrm{q}}}$ and pole $b^{q}{ }_{j}$ are calculated. When $q$ transforms from 0 to $\mathrm{m}$ in turn, the ergodic range of traversal of $b_{j}$ is realized. Finally, select the minimum value of $J_{1 j}{ }^{q}(q=0,1, \cdots, m)$ as $J_{1 j}{ }^{*}$, the corresponding $b^{q}{ }_{j}$ is converted into ${ }^{*}$

When ${ }^{b_{j}}$ is staying in the interval $\left[a_{q}, a_{q+1}\right]$, the detailed steps can be shown as: While $b_{j} \in\left[a_{q} a_{q+1}\right]$, then $a_{0} \leq \cdots \leq a_{q} \leq b_{j} \leq a_{q+1} \leq \cdots \leq a_{m+1}$, then $J_{1 j}=\sum_{i=1}^{q}\left|a_{i}-r_{i j}\right|+\sum_{i=q+1}^{m}\left|b_{j}-r_{i j}\right|$. For the sequence $\left\{r_{i j}^{\prime}\right\}_{i=q+1}^{m}$, it is ordered form small to big as $\left\{r_{i j}^{\prime \prime}\right\}_{i=q+1}^{m}$, we can get: $J_{1 j}=\sum_{i=1}^{q}\left|a_{i}^{\prime}-r_{i j}^{\prime}\right|+\sum_{i=q+1}^{m}\left|b_{j}-r_{i j}^{\prime}\right|$

From the above steps, we can get $B=\left(b_{1}^{*}, \cdots, b_{n}^{*}\right)$, and $J_{1}^{*}=\sum_{\mathrm{j}=1}^{n} J_{1 \mathrm{j}}^{*}$.

\section{Design of fuzzy PID controller for electric vehicle battery power control system}

The optimal fuzzy reasoning method is applied to the fuzzy PID control, the optimal fuzzy reasoning is combined with the traditional PID control. The PID control is referred as the most commonly used controller, there are lots of advantages other controllers cannot match, the structure is simple, the parameter tuning method is relatively mature, and it can be well explained. The proportional part can reflect the current error information, the integral can reflect the error information in the past comprehensively. The differential can reflect the error information next trend. In simple terms, PID controller can reflect the error information of the past, present and future.

The controller of this system tracks the output error $e=y-r$ ( $r$ is the command signal, $y$ is the output signal). The error change rate $e$ is the input vector, $u$ is the intermediate variable, the final control output is $u^{P I D}=K_{0} \int u d t+K_{1} u$. 
The input variables and output variables of electric vehicle battery power controller are discussed, the discourse domain of each variable is $[-1,1]$, a standard triangular membership function is taken, membership is divided into $2 \mathrm{n}+1$, where $\mathrm{n}$ is odd, the two-dimensional linear rule base is shown in Table 1.

Table 1 Two-dimensional linear rule base of power controller variables

\begin{tabular}{cccccccc}
\hline E1 & & & & E2 & & & \\
& & & & & & \\
& NL & MN & NS & ZR & PS & PM & PL \\
\hline PL & ZR & PS & PM & PL & PL & PL & PL \\
PM & NS & ZR & PS & PM & PL & PL & PL \\
PS & NM & NS & ZR & PS & PM & PL & PL \\
ZR & NL & NM & NS & ZR & PS & PM & PL \\
NS & NL & NL & NM & NS & ZR & PS & PM \\
NM & NL & NL & NL & NM & NS & ZR & PS \\
NL & NL & NL & NL & NL & NM & NS & ZR \\
\hline
\end{tabular}

\section{Experiment results and analysis}

Digital simulation is taken in the Matlab environment, and battery power control system of electric vehicle uses the one order inertial link to control lag objects, some time-varying disturbances are added, it is: $G(s)=\frac{75}{63 s+1} e^{-5 s}+f(t)$, classical PID method and new optimal fuzzy PID method are used in the simulation for comparison, simulation results are shown in Figure 1 as follows:

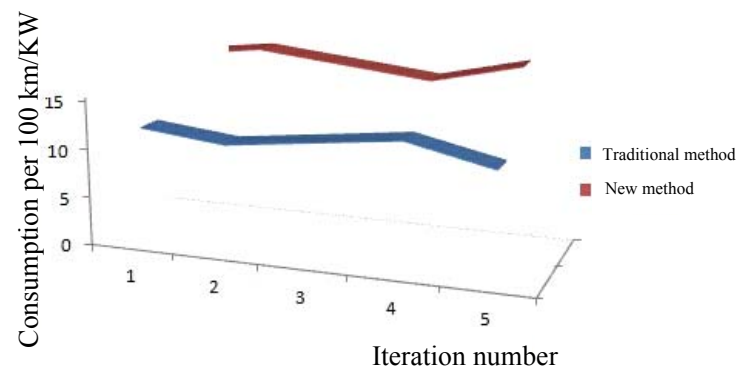

Figure 1. Power control results of different algorithms

From figure 1 as above, we can conclude that new optimal fuzzy PID control algorithm can quickly find the solution of the problem accurately, this algorithm can quickly converge to the global optimal solution, and the desired results are achieved.

\section{Conclusions}

In this paper, the existing electric vehicle battery power control algorithm is improved by the optimal control, the optimal fuzzy algorithm is used in PID controller design, battery electric power control is realized, the multilayer parallel search structure is proposed, and the premature evaluation index is constructed. Finally, the algorithm is used to solve the problem of the global optimal 
convergence solutions. The experiment results show that, this algorithm is used in power control for electric vehicle battery, which can reduce the electric vehicle battery energy consumption, the dilemma of resources and energy shortage can be eased to a certain degree.

\section{Acknowledgement}

Jiangxi Provincial of science and technology support program:

《Research solar charging systems application of electric-vehicle 》

\section{References}

[1] YANG Ming, HAO Liang, XU Dianguo. Online suppression of mechanical resonance b ased on adapting notch filter[J]. Journal of Harbin Institute of Technology, 2014,46(4):63 $-69$.

[2] Zhang Zilong, Xue Jing, Qiao Honghai, et al. The Vehicle Retrieval Methods of Traffi c Video Based on Improved SURF Algorithm[J]. Journal of Northwestern Polytechnical University, 2014,32(2):297-301.

[3] Alfaro V M, Vilanovab R. Robust tuning of 2DoF five-parameter PID controllers for inv erse response controlled processes[J]. Journal of Process Control, 2013,23(4): 453-462.

[4] Liu Xiangdong. Data Clustering Algorithm and Software Design Based on Disturbance S earching of Logistic Series[J]. Bulletin of Science and Technology, 2014,30(2): 161-163

[5] WANG Hai-feng, LU Jun. Research and Simulation on Complex Electric Power Equipme nt Sudden Fault Diagnosis[J]. Computer Simulation, 2013, 30(3): 127-129. 